\title{
Evaluation of homeopathic drugs on Glucocorticoid induced osteoporosis (GIOP) zebrafish model
}

\author{
Himanshu Gupta ${ }^{1}$ and Mansee Thakur ${ }^{2}$ \\ ${ }^{1}$ Department of Medical Genetics, Tutor, MGM School of Biomedical Sciences, MGM Medical College Building, MGM \\ Institute of Health Sciences, Kamothe, Navi Mumbai (M.S.) India \\ ${ }^{2}$ Department of Medical Biotechnology and Central Research Laboratory, MGMCET, MGM School of Biomedical \\ Sciences, MGM Medical College Building, MGM Institute of Health Sciences, Kamothe, Navi Mumbai (M.S.) India \\ Email : mansibiotech79@gmail.com
}

Article Info : Received : 30.01.2018; Revised : 12.03.2018; Accepted : 25.03.2018

Background: Homeopathic remedies are proposed as source for traditional preventive medicines. In this context our study focuses to evaluate efficacy of homoeopathic drug with reference to GIOP in Zebrafish vertebrate model. The overall approach behind this study was to determine whether homeopathic drugs can be used along with of allopathic medicines as an alternate therapy. Methods: In the present study, an attempt is made to find out the efficacy of homoeopathic medicines- Argentum Metallicum, Calcaria Carbonica and Sepia in two dilutions 6CH and $30 \mathrm{CH}$ on GIOP model of zebra fish in two dilutions. Exposure was studied at 3 different points - 4, 14 and 28 days exposure (treatment with test drugs) and staining intensities were measured using Image $\mathbf{J}$ software. Control groups used were untreated control larvae, GIOP model untreated post 11 dpf and GIOP model treated with Alendronate. The medium was changed every day at the same time. Staining intensities measured for Alizarin Red and Calcein dye stained images. Results: Staining intensities of Alizarin red and Calcein staining of the treated group on statistical analysis showed that the means of GIOP and other treated groups were not statistically significant at $\mathrm{p}<0.05$ except in the case of 28 day study for Calcarea Carbonica 30C and Sepia 30C. Though screening was processed using Alizarin red and Calcein staining, our quantification screening with calcein labeling indeed facilitated the process and diminished labor of handling. Conclusion:Our findings show that, despite some physiological differences between mammals and teleosts, the zebra fish represents an effective model for screening of bone defects. This model has been used for preliminary studies on homeopathy drugs prescribed for treatment of Osteoporosis. The proposed calcein staining protocol can represents a powerful tool for in vivo monitoring of mineralized structures.

Key words : Homeopathy, Osteoporosis, Zebra fish, Prednisolone, Alizarin red, Calcein

How to cite this paper : Gupta, Himanshu and Thakur, Mansee (2018). Evaluation of homeopathic drugs on Glucocorticoid induced osteoporosis (GIOP) zebrafish model. Asian J. Bio. Sci., 13 (1) : 32-38.DOI : 10.15740/HAS/AJBS/13.1/32-38. 